\title{
Simultaneous measurement of the neutron capture and fission yields of ${ }^{233} \mathrm{U}$
}

\author{
E. Berthoumieux ${ }^{1, a}$, U. Abbondanno ${ }^{2}$, G. Aerts ${ }^{1}$, H. Álvarez ${ }^{3}$, F. Álvarez-Velarde ${ }^{4}$, S. Andriamonje ${ }^{1}$, J. Andrzejewski $^{5}$, \\ P. Assimakopoulos ${ }^{\dagger 6}$, L. Audouin ${ }^{7}$, G. Badurek ${ }^{8}$, P. Baumann ${ }^{9}$, F. Bečváŕr ${ }^{10}$, F. Calviño ${ }^{11}$, M. Calviani ${ }^{12,13}$, D. Cano-Ott ${ }^{4}$, \\ R. Capote ${ }^{14,15}$, C. Carrapiço ${ }^{1,16}$, P. Cennini $^{17}$, V. Chepe ${ }^{18}$, E. Chiaveri ${ }^{17}$, N. Colonna ${ }^{19}$, G. Cortes ${ }^{20}$, A. Couture $^{21}$, \\ J. Cox $^{21}$, M. Dahlfors ${ }^{17}$, S. David ${ }^{7}$, I. Dillmann ${ }^{22}$, C. Domingo-Pardo ${ }^{22,23}$, W. Dridi ${ }^{1}$, I. Duran ${ }^{3}$, C. Eleftheriadis ${ }^{24}$, \\ M. Embid-Segura ${ }^{4}$, L. Ferrant ${ }^{\dagger 7}$, A. Ferrari ${ }^{17}$, R. Ferreira-Marques ${ }^{18}$, K. Fujii ${ }^{2}$, W. Furman ${ }^{25}$, I. Goncalves ${ }^{18}$, \\ E. González-Romero ${ }^{4}$, F. Gramegna ${ }^{12}$, C. Guerrero ${ }^{4}$, F. Gunsing ${ }^{1}$, B. Haas ${ }^{26}$, R. Haight ${ }^{27}$, M. Heil ${ }^{22}$, A. Herrera-Martinez ${ }^{17}$, \\ M. Igashira ${ }^{28}$, E. Jericha ${ }^{8}$, F. Käppeler ${ }^{22}$, Y. Kadi ${ }^{17}$, D. Karadimos ${ }^{6}$, D. Karamanis ${ }^{6}$, M. Kerveno ${ }^{9}$, P. Koehler ${ }^{29}$, \\ E. Kossionides ${ }^{30}$, M. Krtička ${ }^{10}$, C. Lampoudis ${ }^{1,24}$, H. Leeb ${ }^{8}$, A. Lindote ${ }^{18}$, I. Lopes ${ }^{18}$, M. Lozano ${ }^{15}$, S. Lukic ${ }^{9}$, \\ J. Marganiec ${ }^{5}$, S. Marrone ${ }^{19}$, T. Martínez ${ }^{4}$, C. Massimi ${ }^{31}$, P. Mastinu ${ }^{12}$, A. Mengoni ${ }^{14,17}$, P.M. Milazzo ${ }^{2}$, C. Moreau ${ }^{2}$, \\ M. Mosconi ${ }^{22}$, F. Neves ${ }^{18}$, H. Oberhummer ${ }^{8}$, S. O’Brien ${ }^{21}$, J. Pancin ${ }^{1}$, C. Papachristodoulou ${ }^{6}$, C. Papadopoulos ${ }^{32}$, \\ C. Paradela ${ }^{3}$, N. Patronis ${ }^{6}$, A. Pavlik ${ }^{33}$, P. Pavlopoulos ${ }^{34}$, L. Perrot ${ }^{1}$, M.T. Pigni ${ }^{8}$, R. Plag ${ }^{22}$, A. Plompen ${ }^{35}$, A. Plukis ${ }^{1}$, \\ A. Poch $^{20}$, J. Praena ${ }^{12}$, C. Pretel ${ }^{20}$, J. Quesada ${ }^{15}$, T. Rauscher ${ }^{36}$, R. Reifarth ${ }^{27}$, C. Rubbia ${ }^{37}$, G. Rudolf ${ }^{9}$, P. Rullhusen ${ }^{35}$, \\ J. Salgado ${ }^{16}$, C. Santos ${ }^{16}$, L. Sarchiapone ${ }^{17}$, I. Savvidis ${ }^{24}$, C. Stephan ${ }^{7}$, G. Tagliente ${ }^{19}$, J.L. Tain ${ }^{23}$, L. Tassan-Got ${ }^{7}$, L. Tavora $^{16}$, \\ R. Terlizzi ${ }^{19}$, G. Vannini ${ }^{31}$, P. Vaz ${ }^{16}$, A. Ventura ${ }^{38}$, D. Villamarin ${ }^{4}$, M.C. Vincente ${ }^{4}$, V. Vlachoudis ${ }^{17}$, R. Vlastou $^{32}$, \\ F. Voss ${ }^{22}$, S. Walter ${ }^{22}$, M. Wiescher ${ }^{21}$, and K. Wisshak ${ }^{22}$
}

The n_TOF Collaboration (www.cern.ch/ntof)

${ }^{1}$ CEA/Saclay-DSM/DAPNIA, Gif-sur-Yvette, France $-{ }^{2}$ Istituto Nazionale di Fisica Nucleare, Trieste, Italy $-{ }^{3}$ Universidade de Santiago de Compostela, Spain $-{ }^{4}$ Centro de Investigaciones Energeticas Medioambientales y Tecnologicas, Madrid, Spain - ${ }^{5}$ University of Lodz, Lodz, Poland $-{ }^{6}$ University of Ioannina, Greece $-{ }^{7}$ Centre National de la Recherche Scientifique/IN2P3-IPN, Orsay, France $-{ }^{8}$ Atominstitut der Österreichischen Universitäten, Technische Universität Wien, Austria $-{ }^{9}$ Centre National de la Recherche Scientifique/IN2P3-IReS, Strasbourg, France $-{ }^{10}$ Charles University, Prague, Czech Republic $-{ }^{11}$ Universidad Politecnica de Madrid, Spain $-{ }^{12}$ Istituto Nazionale di Fisica Nucleare, Laboratori Nazionali di Legnaro, Italy $-{ }^{13}$ Dipartimento di Fisica, Università di Padova, Italy $-{ }^{14}$ International Atomic Energy Agency (IAEA), Nuclear Data Section, Vienna, Austria $-{ }^{15}$ Universidad de Sevilla, Spain $-{ }^{16}$ Instituto Tecnológico e Nuclear (ITN), Lisbon, Portugal $-{ }^{17}$ CERN, Geneva, Switzerland $-{ }^{18}$ LIP-Coimbra \& Departamento de Fisica da Universidade de Coimbra, Portugal - ${ }^{19}$ Istituto Nazionale di Fisica Nucleare, Bari, Italy $-{ }^{20}$ Universitat Politecnica de Catalunya, Barcelona, Spain $-{ }^{21}$ University of Notre Dame, Notre Dame, USA - ${ }^{22}$ Forschungszentrum Karlsruhe GmbH (FZK), Institut für Kernphysik, Germany - ${ }^{23}$ Instituto de Física Corpuscular, CSICUniversidad de Valencia, Spain $-{ }^{24}$ Aristotle University of Thessaloniki, Greece - ${ }^{25}$ Joint Institute for Nuclear Research, Frank Laboratory of Neutron Physics, Dubna, Russia $-{ }^{26}$ Centre National de la Recherche Scientifique/IN2P3-CENBG, Bordeaux, France $-{ }^{27}$ Los Alamos National Laboratory, New Mexico, USA - ${ }^{28}$ Tokyo Institute of Technology, Tokyo, Japan - ${ }^{29}$ Oak Ridge National Laboratory, Physics Division, Oak Ridge, USA $-{ }^{30}$ NCSR, Athens, Greece $-{ }^{31}$ Dipartimento di Fisica, Università di Bologna, and Sezione INFN di Bologna, Italy $-{ }^{32}$ National Technical University of Athens, Greece $-{ }^{33}$ Institut für Isotopenforschung und Kernphysik, Universität Wien, Austria - ${ }^{34}$ Pôle Universitaire Léonard de Vinci, Paris-La Défense, France $-{ }^{35}$ CEC-JRC-IRMM, Geel, Belgium - ${ }^{36}$ Department of Physics-University of Basel, Switzerland $-{ }^{37}$ Università degli Studi Pavia, Pavia, Italy $-{ }^{38}$ ENEA, Bologna, Italy

\begin{abstract}
We have measured the neutron capture and fission cross section of ${ }^{23} \mathrm{U}$ at the neutron time-of-flight facility $\mathrm{n}_{-} \mathrm{TOF}$ at CERN in the energy range from $1 \mathrm{eV}$ to $1 \mathrm{MeV}$ with high accuracy by using a high performance $4 \pi \mathrm{BaF}_{2}$ Total Absorption Calorimeter (TAC) as a detection device. The method, based on the TAC energy response study, allowing to disentangle between $\gamma$ 's originating from fission and capture will be presented as well as the first very preliminary results.
\end{abstract}

\section{Introduction}

The use of a fuel cycle based on ${ }^{232} \mathrm{Th}$ could substantially reduce the radiotoxicity of the produced nuclear waste, since the lower atomic and mass numbers of thorium results in a strongly suppressed build-up of the higher actinides, especially americium and curium. The design and realization of nuclear power stations based on the use of thorium require an

${ }^{a}$ Presenting author, e-mail: Eric.Berthoumieux@cea.fr accurate knowledge of the neutron reactions cross section of ${ }^{233} \mathrm{U}$, since this isotope plays the same role as ${ }^{235} \mathrm{U}$ in standard nuclear power plants.

The neutron capture reaction cross section measurement of ${ }^{233} \mathrm{U}$, is complicated by low energy neutron induced fission that will also give $\gamma$-rays. Up to now only two measurements are available in the resonance region. The first one performed by Brooks et al. [1] up to $10 \mathrm{eV}$ with a poor energy resolution. Another measurement has been performed by Weston et al. [2] up to $1 \mathrm{keV}$ using the fission tagging technique. A similar technique is being developed at LANSCE using DANCE $[3,4]$ but no measurement on ${ }^{233} \mathrm{U}$ has been reported. 


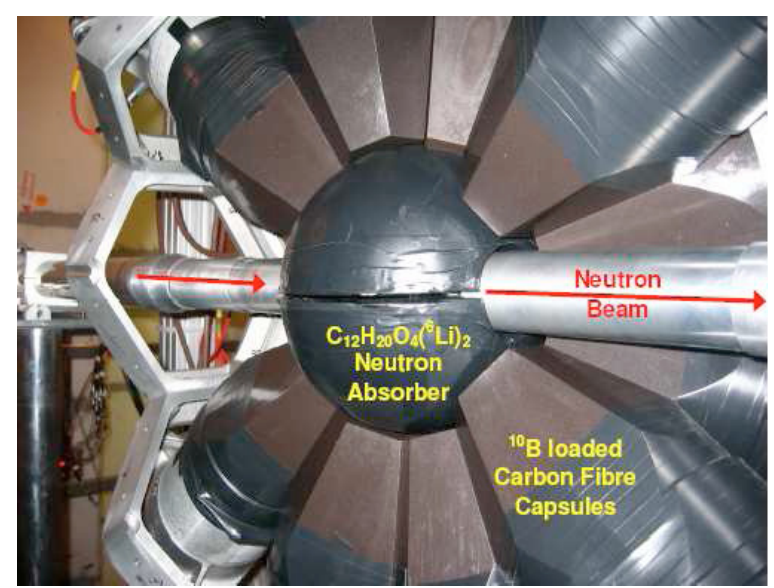

Fig. 1. Picture of one hemisphere of the $n_{-}$TOF TAC. The picture depicts the neutron beam pipes, the central neutron absorber, and the ${ }^{10} \mathrm{~B}$ loaded carbon fiber capsule.

For these reasons, we have measured simultaneously the neutron capture and fission cross section of ${ }^{233} \mathrm{U}$ at the neutron time-of-flight facility, n_TOF, at CERN [5] by studying the calorimetric response of the newly constructed n_TOF Total Absorption Calorimeter (TAC) [6,7].

\section{Experimental set-up}

The $\mathrm{n}_{-}$TOF facility at CERN is a neutron source with a wide energy range, where neutrons are generated by spallation induced by $20 \mathrm{GeV} / \mathrm{c}$ protons onto a solid lead target. The proton beam has a time resolution of $6 \mathrm{~ns}$ (r.m.s.) and an intensity up to $7 \cdot 10^{12}$ protons/pulse. The flight distance of about $185 \mathrm{~m}$, combined with the very high instantaneous flux, allows to perform high resolution measurements on radioactive target.

The sample of $108 \mathrm{mg}$ of $\mathrm{U}_{3} \mathrm{O}_{8}$ (91 mg of $\mathrm{U}, 0.356 \mathrm{MBq}$ ) was pressed into a pellet and doubly encapsulated between $\mathrm{Al}$ and $\mathrm{Ti}$ foils which were $0.15 \mathrm{~mm}$ and $0.2 \mathrm{~mm}$ thick respectively, in order to fulfill the ISO 2919 certification requested by the safety regulations at CERN. It isotopic purity, determined by $\gamma$-ray spectroscopy was: ${ }^{233} \mathrm{U} 99.01 \%,{ }^{234} \mathrm{U} 0.74 \%$, ${ }^{235} \mathrm{U} 0.23 \%,{ }^{238} \mathrm{U} 0.04 \%$. During our analysis only ${ }^{234} \mathrm{U}$ contribution has been seen and subtracted.

The design of the $\mathrm{n}_{-} \mathrm{TOF}$ Total Absorption Calorimeter (TAC) $[6,7]$ is based on $40 \mathrm{BaF}_{2}$ crystals shaped as hexagonal and pentagonal pyramids forming a spherical shell with $10 \mathrm{~cm}$ inner radius and $15 \mathrm{~cm}$ thickness. Each $\mathrm{BaF}_{2}$ is covered by a teflon light reflector layer, an aluminum foil and a final cover with ${ }^{10} \mathrm{~B}$ loaded carbon fiber capsule. The TAC has a high detection efficiency for capture events near $100 \%$ and an acceptable energy resolution, typically $10 \%$ at $661.5 \mathrm{keV}$ to $6 \%$ at $6.15 \mathrm{MeV}$. Targets are placed at the center of the TAC and surrounded by a neutron absorber made of $\mathrm{C}_{12} \mathrm{H}_{20} \mathrm{O}_{4}\left({ }^{6} \mathrm{Li}\right)_{2}$. The neutron absorber as the ${ }^{10} \mathrm{~B}$ loaded carbon fiber reduce the sensitivity of the detector to neutron without affecting too much the capture detection efficiency.

In between two energy calibration, the energy drift is monitored by using the $\alpha$ intrinsic radioactivity of $\mathrm{BaF}_{2}$ crystals.

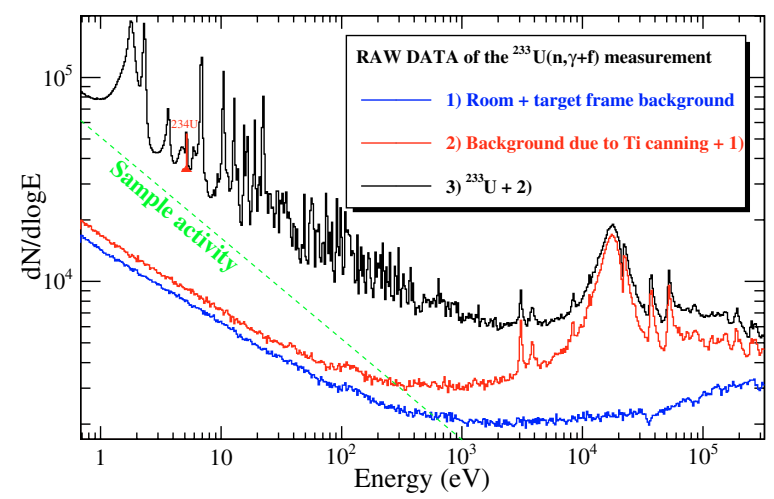

Fig. 2. Raw data of ${ }^{233} \mathrm{U}(\mathrm{n}, \gamma+\mathrm{f})$ measurement illustrating the various contributions to the signal.

\section{Data reduction}

In order to improve the signal to background ratio, one first needs to determine an optimal event selection criteria for the TAC. In our case, as can be seen in figure 2 , the main sources of background are: the sample radioactivity below $\approx 100 \mathrm{eV}$ and the scattered neutrons above this energy although the sample is surrounded by neutron absorber. This is because our samples were encapsulated in between two titanium foils, as describe before, in order to be ISO-2919 compliant. In order to minimize this component we kept only events for which more than one cluster is present, where a cluster consists of a connection of neighboring crystals that have been hitted.

In order to disentangle betwen all signals contributions, one performs a shape analysis of the TAC energy response for each time-of-flight (neutron energy) bin. The method is similar to the one used for the ${ }^{234} \mathrm{U}$ neutron capture expriment $[8,9]$. Nevertheless an additional step is needed in this case, since we need to disentangle in between the TAC response to neutron captured by a ${ }^{233} \mathrm{U}$ nucleus, to the one given by the fission process.

The top left part of figure 3 shows the fission (magenta) and capture yield (blue) corresponding to our target given by ENDF/B-VII [10] from 1 to $10 \mathrm{eV}$ incoming energy. Around $4.5 \mathrm{eV}$ one can see a resonance in fission, not present in capture. The bottom left part of figure 3 shows the TAC energy response (in black) of this resonance selected as shown in the top right part of figure 3. The fission only response, in red in the left bottom part, has been obtained by subtracting the TAC response in the vicinity of the resonance.

By selecting another resonance, and applying the same procedure one can obtain the TAC response to capture and fission given by the red curve in the right bottom part of figure 3. Considering that the signal above $8 \mathrm{MeV}$ can only be attributed to fission $\left(S_{n}=6.845 \mathrm{MeV}\right.$ for ${ }^{233} \mathrm{U}$ ) one can easily subtract the previously obtained fission response (in blue) to obtain the TAC response to capture only (yellow area).

The next step of the data reduction is the efficiency determination, that also take care of the "software" dead time $[8,9]$. For the capture part a simulation, that consists in a $\gamma$-ray cascade generator [11] coupled to the GEANT4 [12] toolkit, has been performed. For fission no TAC efficiency has been yet 

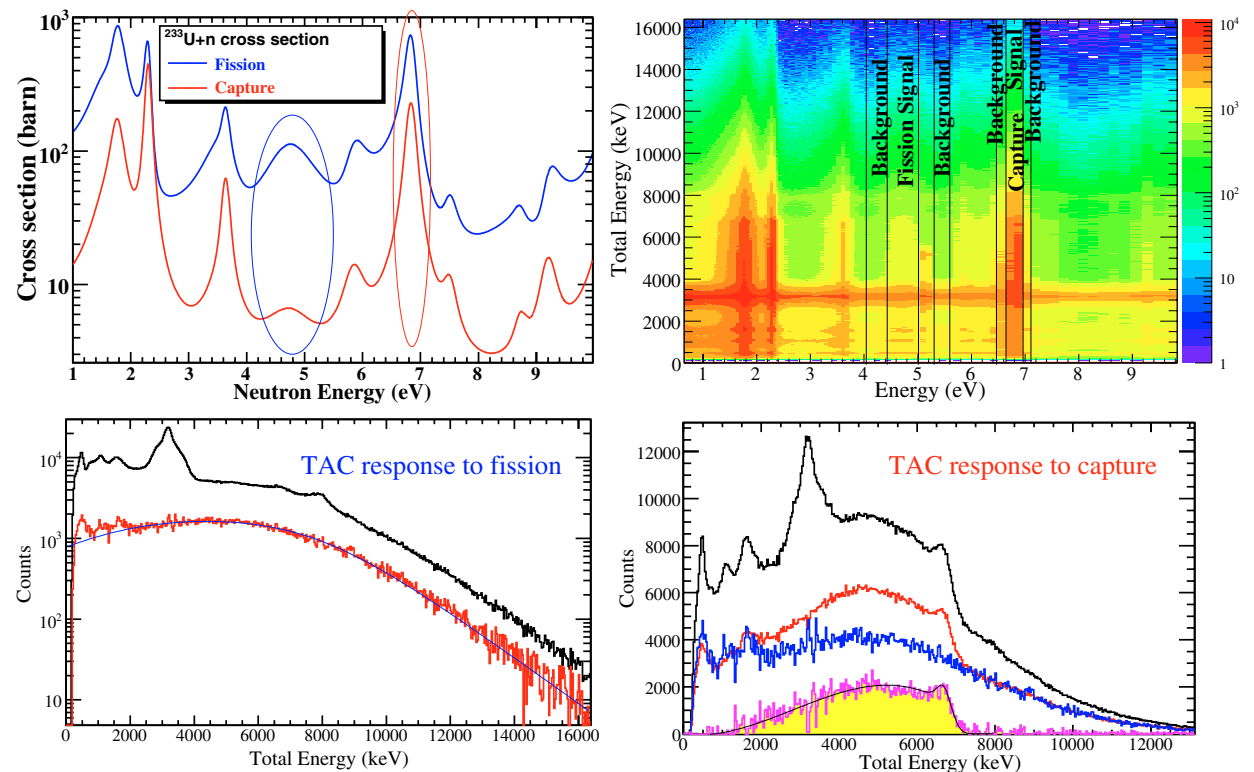

Fig. 3. Isolating the TAC response to fission and capture (see text).

determined. A $100 \%$ efficiency is considered for the present preliminary results.

The data, converted from time-of-flight to neutron incoming energy assuming a flight path length of $184.9 \pm 0.1 \mathrm{~m}$, are then divided by the $n_{-}$TOF standard flux times the beam fraction seen by the sample to obtain yields.

Finally the capture and fission yields are converted to cross section assuming a ${ }^{233} \mathrm{U}$ thickness of $2.99410^{-4}$ at/b. Data have been corrected for self shielding effect by taking elastic cross section from ENDF/B-VII [10] evaluation.

\section{Preliminary results}

Because at the present stage, the fission efficiency and the ${ }^{233} \mathrm{U}$ mass distribution inside the canning is not known with a sufficient precision, our fission cross section has been scaled to reproduce the ENDF/B-VII evaluation below $20 \mathrm{eV}$ by a factor of $0.91 \pm 0.01$. This factor has also been taken into account to derive the capture cross section.

Figure 4 shows a comparison between our scaled fission cross section (in black) and the ones given by Weston et al. [2] (in red) measured at RPI facility, Cao et al. [13] (in cyan) measured at GELINA, Guber et al. [14] (in blue) recently measured at ORELA, Calviani et al. [15] (in magenta) recently measured at CERN by using a fission chamber, togheter with the latest ENDF/B-VII [10] (in green) evaluation, for neutrons incoming energy below $20 \mathrm{eV}$. The overall agreement between all datasets is excellent. Guber's cross section for the first resonance is slightly below all measurements, but this can be easily explained because this dataset has not been corrected for self-shielding.

To compare the smooth evolution of each dataset with energy, all datasets have been rebinned to 20 bins/decade in energy ( 0.05 binwidth in lethargy units). The results is shown on figure 5 for neutron incoming energies from $0.5 \mathrm{eV}$ to $3 \mathrm{keV}$.

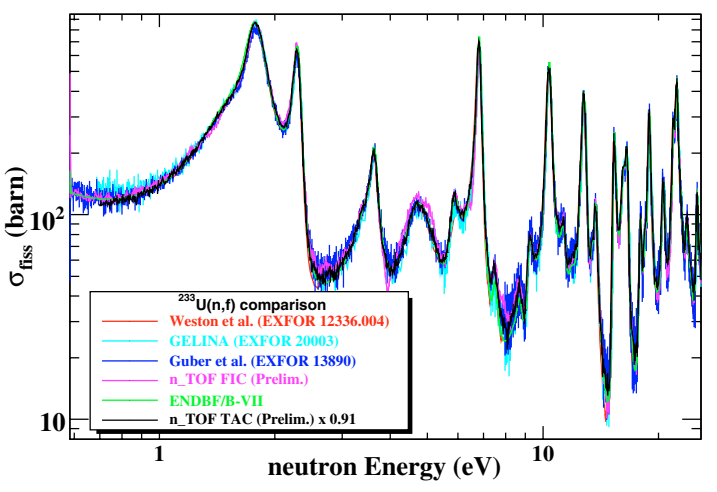

Fig. 4. Comparison of various measurements of neutron induced fission cross section of ${ }^{233} \mathrm{U}$ in the energy range $1-20 \mathrm{eV}$.

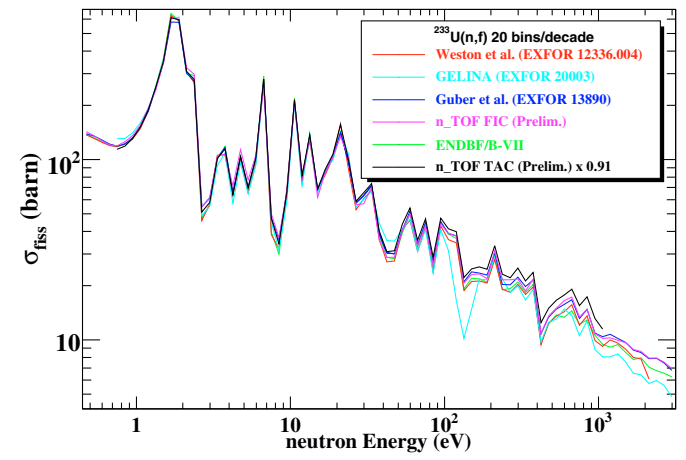

Fig. 5. Comparison of various measurements of neutron induced fission cross section of ${ }^{233} \mathrm{U}$ up to $3 \mathrm{keV}$.

Below $100 \mathrm{eV}$ all measurements are compatible within $10 \%$. One can see a perfect agreement between Guber's data [14] and Calviani's data [15] in the energy range of interest but slighly above Weston's [2] and ENDF/B-VII [10] for neutrons 


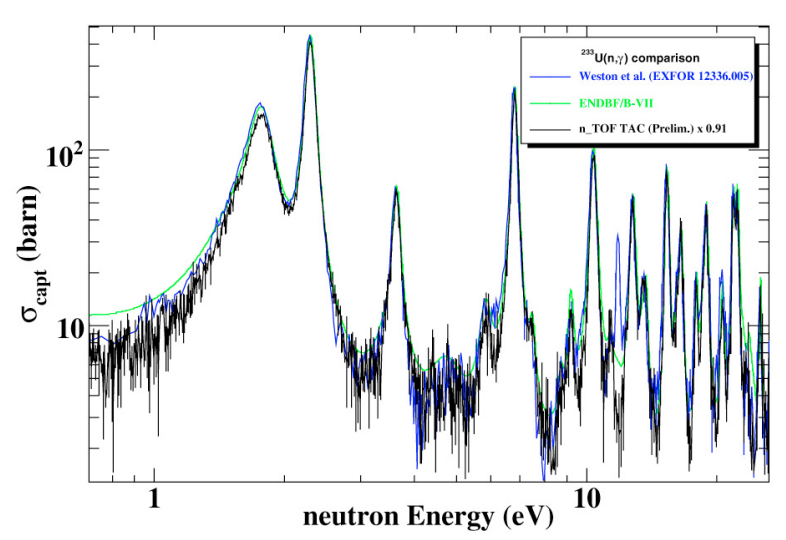

Fig. 6. Comparison of various measurements of neutron capture cross section of ${ }^{233} \mathrm{U}$ in the energy range $1-20 \mathrm{eV}$.

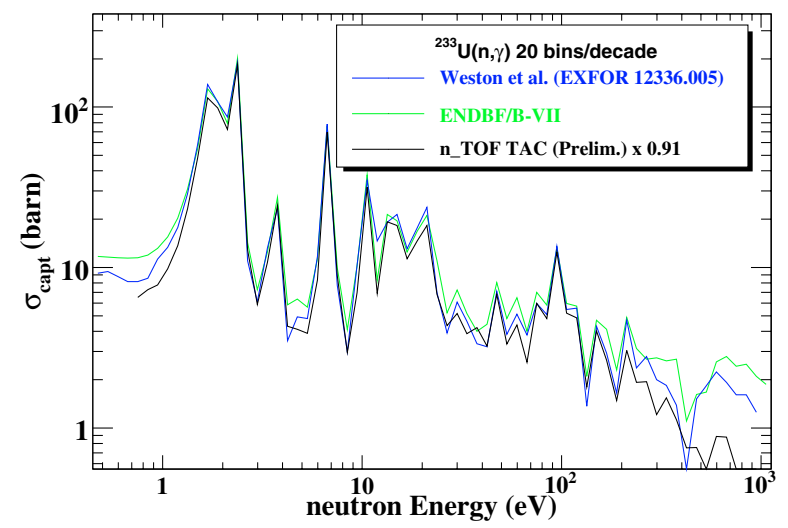

Fig. 7. Comparison of various measurements of neutron capture cross section of ${ }^{233} \mathrm{U}$ up to $1 \mathrm{keV}$.

energies above $\approx 100 \mathrm{eV}$. On the contrary Cao's data [13] above around $500 \mathrm{eV}$ gives the lowest cross section. The discrepants points around $100 \mathrm{eV}$ in Cao's data are due to the presence of a black resonance. Finally our data starts to overestimate all other datasets by about $10 \%$ starting from $100 \mathrm{eV}$. This could be due to a slight change of TAC energy response to fission with increasing energy not considered in the present analysis.

Figure 6 shows a comparison between our scaled capture cross section (in black) and the ones given by Weston et al. [2] (in blue) measured at RPI facility togheter with the latest ENDF/B-VII [10] (in green) evaluation, for neutrons incoming energy below $20 \mathrm{eV}$. One can see a good agreement in shape between our data and Weston's data, except for the first resonance where our contribution is about $10 \%$ lower than Weston. A resonance is also seen in Weston data around $11 \mathrm{eV}$, but not in our data neither reported in the evaluation. Our resonances also shows a deeper fall-off on the low energy side that could be attributed to the better energy resolution of the $\mathrm{n}_{-}$TOF facility compared to the RPI facility.

As for fission cross section measurements, capture datasets have been rebinned to exhibit the slow evolution with incoming neutrons energies. The result is shown on figure 7 . The agreement with Weston's data is good from $2 \mathrm{eV}$ to $200 \mathrm{eV}$ even though our data seems in average lower by a few percent. Above $200 \mathrm{eV}$ our data starts to deviate from Weston's measurements. This problem may be linked to our possibly overestimation of fission cross section above this energy as seen before.

Nevertheless since our fission detection efficiency is surestimated, for sure, one can infer that the capture cross section given by Weston [2] is probably overestimated by up to $20 \%$ in neutrons energies from 0.5 to $200 \mathrm{eV}$.

\section{Conclusion}

An innovative technique, based on a calorimetric shape analysis, to disentangle between capture and fission induced by neutrons, has been presented. This technique has been employed to extract the neutron capture and fission cross section of a ${ }^{233} \mathrm{U}$ measurement performed using the Total Absorption Calorimeter (TAC) at n_TOF at CERN. Combining this technique with fission tagging should allow to determine capture cross section of fissionable isotope with an unprecedent precision.

We acknowledge the CERN participation in the $\mathrm{n}_{-} \mathrm{TOF}$ project. This work has been supported by the European Commission's $5^{\text {th }}$ Framework Programme under contract FIKW-CT-2000-00107.

\section{References}

1. F. Brooks, J. Joly, M. Schomberg, M. Sowerby, Tech. Rep. AERE-M-1709, United Kingdom Atomic Energy Research Establisment, Harwell, Berks., England (1966).

2. L. Weston, R. Gwin, G. de Saussure, R. Fullwood, R. Hockenbury, Nucl. Sci. Eng. 34, 1 (1968).

3. T. Bredeweg et al., Simultaneous measurement of $(n, \gamma)$ and (n,fission) cross sections with the DANCE $4 \pi \mathrm{BaF}_{2}$ array, in $12^{\text {th }}$ Int. Symp. on Capture gamma-ray spectroscopy and related topic (AIP Conference Proceedings, Notre Dame, USA, 2005), Vol. 819, pp. 568-569.

4. T. Bredeweg et al., Recent actinide nuclear data efforts with the DANCE $4 \pi \mathrm{BaF}_{2}$ array (these proceedings).

5. C. Rubbia et al., CERN CERN-LHC-98-02 (EET), CERN, Genève (1998).

6. K. Wisshak et al., Nucl. Instrum. Meth. A 292, 595 (1990).

7. M. Heil et al., Nucl. Instrum. Meth. A 459, 229 (2001).

8. W. Dridi et al., Measurement of the neutron capture cross section of ${ }^{234} U$ in $n_{-}$TOF at CERN, in PHYSOR-2006 ANS Topical Meeting on Reactor Physics (Vancouver, BC, Canada, 2006).

9. W. Dridi, Ph.D. thesis, Université d'Évry Val d'Essonne (2006).

10. M. Chadwick et al., Nucl. Data Sheets 107, 2931 (2006).

11. J. Tain, F. Gunsing, D. Cano-Ott, Monte carlo generation of capture cascades, Presentation at $7^{\text {th }} \mathrm{n}_{-}$TOF Meeting (2000).

12. S. Agostinelli et al., Nucl. Instrum. Meth. A 506, 250 (2003).

13. M. Cao, E. Migneco, J. Theobald, M. Merla, J. Nucl. Energy 24, 111 (1970).

14. K. Guber, R. Spencer, L. Leal, J. Harvey, N. Hill, G. Dos Santos, R. Sayer, D. Larson, Nucl. Sci. Eng. 135, 141 (2000).

15. M. Calviani et al., Measurement of the neutron induced fission of ${ }^{235} \mathrm{U},{ }^{233} \mathrm{U}$ and ${ }^{245} \mathrm{Cm}$ with the FIC detector at CERN $n_{-} T O F$ facility (these proceedings). 